\title{
Hull Form Optimization in Waves Based on CFD Technique
}

\author{
Zhang Shenglong ${ }^{1}$, Tahsin Tezdogan ${ }^{2}$, Zhang Baoji ${ }^{3}$, Xu Leping $^{1}$, Lai Yuyang ${ }^{4}$ \\ (1. Shanghai Maritime University, Merchant Marine College, Shanghai 201306, China; 2. \\ University of Strathclyde, Department of Naval Architecture, Ocean and Marine Engineering, \\ Glasgow G4 OLZ, UK; 3. Shanghai Maritime University, College of Ocean Environment and \\ Engineering, Shanghai 201306, China; 4. Beijing Soyotec Co., Ltd., Beijing 100062, China)
}

\begin{abstract}
The seakeeping behavior of a ship in waves is different from its behavior in calm water. The resistance and seakeeping performance of a ship are of great importance and must be considered in the early-stage design of a ship's hull form design. Therefore, this paper proposes a hull form optimization framework aiming to achieve the minimum total resistance in waves using a CFD technique. A sinusoidal wave is adopted to establish the numerical wave tank and the overset mesh technique is used to facilitate the motions of the ships in question. Considering the motions of pitching and heaving, the total resistance of the hull in waves is regarded as the objective function which is calculated using the Reynolds averaged Navier-Stokes (RANS) method. The arbitrary shape deformation (ASD) technique is used to change the geometry. Under displacement and design variables, a hybrid algorithm is developed to evaluate the objective function combining the optimal Latin hypercube design (Opt LHD) and the non-linear programming by quadratic Lagrangian (NLPQL) algorithm. Finally, two examples of hull form optimization are presented and discussed for David Taylor Model Basin (DTMB) model 5512 and WIGLEY III cases. The results show the effectiveness of the optimization framework developed in the present study, which can lay the foundation for further optimization of full-scale ships.
\end{abstract}

Keywords: Hull form optimization; CFD; numerical wave tank; ASD technique; hybrid algorithm

\section{Introduction}

Nowadays, with the increase in international trade and the expanding world economy, the rise in use of large and super-large ships has caused increasing fuel consumption and carbon dioxide emissions. To solve this problem, one of the most effective measures is to reduce hull resistance. So far, many designers have used different methods to explore low resistance and energy-saving ships. Park and Choi (2013) built a minimum resistance design procedure for a Series 60 ship using a sequential quadratic programming (SQP). The frictional resistance coefficient was estimated by the ITTC 1957 model-ship correlation line formula and the wave-making resistance coefficient was evaluated by the potential-flow panel method with the non-linear free surface boundary conditions. Zhang and Zhang (2015) developed a minimum total resistance hull form optimization method based on potential flow theory of wave-making resistance and considered the effects of tail viscous separation. Sun et al. (2016) presented a trim optimization approach on a 4250-TEU container ship using the ANSYS FLUENT CFD software package in conjunction with towing tank tests in order to study the influence of trim on the ship's resistance. Mahmood and Huang (2012) developed an optimization loop of a Series 60 hull with the integration of an additive bulb to the parent hull for reducing the total resistance, using the FLUENT software as a tool to evaluate the objective function and a genetic algorithm as an optimization tool. The potential flow and the Reynolds averaged Navier-Stokes (RANS) solvers were used to calculate the calm water resistance of the David Taylor Model Basin (DTMB) model 5415 model at $F r=0.25$, and Serani et al. (2016) developed an optimization framework using dividing rectangles (DIRECT) and deterministic particle swarm optimization (DPSO) algorithms to find the best ship, respectively.

As can be seen, in all cases above, the optimization loops were based on the assumption that the optimum hull is found in calm water, ignoring the influence of waves. However, rough sea conditions can affect ship motions and increase a ship's resistance. Therefore, the seakeeping performance of a ship should be taken into consideration during the preliminary design stage. Kükner and Sariöz (1995) and Özüm et al. (2005) investigated the effects of some specific parameters on the seakeeping 
performance including the main dimensions and hull form parameters using a high speed displacement type vessel and a fast ship, respectively. Kim et al. (2010) developed a multi-objective genetic algorithm to optimize the DTMB5415 ship for reducing resistance and improving seakeeping at the early design stage. A Neumann-Michell (NM) theory was used to calculate the resistance of a ship which was implemented on a practical design-oriented CFD tool, and Bales' (Bales, 1980) seakeeping ranking method was introduced to calculate the seakeeping of a ship. Grigoropoulos and Chalkias (2010) developed a formal methodology for the hull form optimization in calm and rough water. The Rankine source panel method and strip theories were used for the hydrodynamic evaluation. Gammon (2011) developed a fishing boat optimization loop using a multi-objective genetic algorithm (MOGA). The optimization used three performance indices for resistance, seakeeping and stability to obtain optimal hull offsets as well as optimal values for the principal parameters of length, beam and draft. Herein, wave resistance was calculated using a transom modified Michell integral method. Huang et al. (2015) proposed a new optimization procedure with radial basis function (RBF) surrogate model. In the optimization framework, a practical steady ship flow solver (SSF) based on Neumann-Michell $(\mathrm{NN})$ theory and a ship motion program (SMP) based on strip theory were employed to evaluate the drag and the seakeeping performance of the sampling hull forms, respectively. With rapid developments in computer techniques, CFD has become the go-to technique in the simulation of ship hydrodynamic performance and in the calculation of hull resistance in waves (Özdemir et al., 2016; Shen et al., 2014; Carrica et al., 2011; Tezdogan et al., 2015). However, to the best of our knowledge, there is no specific CFD-based study on hull form optimization in waves using the RANS method. To fill this gap, a practical RANS-based ship hull form optimization loop has been developed in waves by integrating the arbitrary shape deformation (ASD) technique to the optimization model to optimize hull forms for reducing the total resistance in waves.

In recent years, many optimization algorithms have been developed, mainly divided into two major categories: gradient optimization algorithms and global optimization algorithms. Due to the diversity of optimization algorithms, each different optimization algorithm has its own advantages and limitations. In order to improve the search capability, convergence speed and reduce calculation time, many improved optimization algorithms have been put forward. A time, cost and load balance-enhanced ant colony optimization (TCLB-EACO) was developed to improve the resource allocation efficiency of cloud computing (Nie et al., 2016). The particle swarm without velocity equation (PSWV) equation optimization algorithm was developed and studied by Tungadio et al. (2016) in the power system state estimation. Genetic algorithm and non-linear programming were combined to achieve the optimization of hull form by Zhang (2012). Recently, one of the optimization techniques widely used is the sequential quadratic programming (SQP) (Rao, 1999), which is the gradient-based optimization algorithm (Park et al., 2015). If the design space is continuous and unimodal, it can search the results along the steepest descent direction quickly. Although it is fairly efficient, it is highly dependent on the initial point, and it is also likely to fall into a local solution. For this reason, a hybrid algorithm has been developed by combining the optimal Latin hypercube design (Opt LHD) and the non-linear programming by quadratic Lagrangian (NLPQL) algorithm (the modified version of the SQP algorithm), and its performance has also been assessed and compared with the original NLPQL algorithm.

The aim of this paper is to describe the development of an optimization approach based on the CFD software STAR-CCM+. Optimization is carried out at design speed. The ASD technique is used to change the geometry, with the overset mesh technique used to facilitate ship motions. The first order wave in STAR-CCM+ is adopted to establish the numerical wave tank. The dynamic fluid body interaction (DFBI) analysis model is used to simulate the ship motion responses and RANS equation solvers are used to solve the flow field and calculate the total resistance of the hull in waves. The hybrid optimization algorithm is developed to evaluate the objective function. Next, two examples of hull form optimization are presented for a model-scale. Finally, the results for the original and optimized hulls are compared.

This paper is organized as follows: Section 2 presents the numerical modeling approaches, including the governing equations, physical modeling, numerical wave tank, boundary conditions of the computational domain, and mesh generation. Section 3 shows the main optimization methodology: 
geometric reconstruction, optimizer, and optimization loop. Following this, in Section 4, two optimization examples are studied and discussed. Finally, the results of this study are briefly summarized in Section 5, along with the recommendation of the future research.

\section{Numerical modeling}

\subsection{Governing equations}

The governing equations for the incompressible turbulent flow are the continuity equation and the RANS equation. These equations can be expressed as:

$$
\begin{gathered}
\frac{\partial U_{i}}{\partial x_{i}}=0 \\
\rho \frac{\partial U_{i}}{\partial t}+\rho U_{j} \frac{\partial U_{i}}{\partial x_{j}}=-\frac{\partial \hat{p}}{\partial x_{i}}+\frac{\partial}{\partial x_{j}}\left(\mu \frac{\partial U_{i}}{\partial x_{j}}-\rho \overline{u_{i} u_{j}}\right)+f_{i}^{*}
\end{gathered}
$$

Where $U_{i}=(U, V, W)$ is the velocity component in the $x_{i}=(x, y, z)$ direction, and $\rho, \hat{p}, \mu$, $-\rho \overline{u_{i} u_{j}}$ and $f_{i}^{*}$ are the fluid density, static pressure, fluid viscosity, Reynolds stresses and body forces per unit volume, respectively.

\subsection{Physical modeling}

\subsubsection{Turbulence model}

Yousefi et al. (2014) note that the Re-Normalization Group (RNG) model has a significant improvement over the standard model, especially where the streamlines are highly curved and thus vortices and circulations exist. In flows with reduced velocity and separation due to a reversed pressure gradient, the RNG model performs better than the standard $k-\varepsilon$ model. Therefore, the RNG $k-\varepsilon$ model (Yakhot \& Orszag, 1986) is selected as a turbulence model to simulate the performance of a ship in waves. The transport equations can be written as follows:

$$
\begin{gathered}
\rho \frac{d k}{d t}=\frac{\partial}{\partial x_{j}}\left[\left(\alpha_{k} \mu_{e f f}\right) \frac{\partial k}{\partial x_{j}}\right]+G_{k}+G_{b}-\rho \varepsilon-Y_{M} \\
\rho \frac{d \varepsilon}{d t}=\frac{\partial}{\partial x_{j}}\left[\left(\alpha_{\varepsilon} \mu_{e f f}\right) \frac{\partial \varepsilon}{\partial x_{j}}\right]+C_{1 \varepsilon} \frac{\varepsilon}{k}\left(G_{k}+C_{3 \varepsilon} G_{b}\right)-C_{2 \varepsilon} \rho \frac{\varepsilon^{2}}{k}-R
\end{gathered}
$$

Where $k$ is the turbulence kinetic energy, $\varepsilon$ is the turbulence dissipation rate, $\mu_{e f f}$ is the effective dynamic viscosity, $G_{k}$ is the generation of turbulent kinetic energy by the mean velocity gradients, $G_{b}$ is the generation of turbulent kinetic energy by buoyancy, $Y_{M}$ represents the contribution of the fluctuating dilatation in compressible turbulence, and $C_{1 \varepsilon}, C_{3 \varepsilon}$ and $C_{2 \varepsilon}$ are the empirical constants.

\subsubsection{Volume of fluid method}

The Volume of Fluid (VOF) method is a surface tracking method fixed under the Euler grid and simulates the multiphase flow model by solving the momentum equation and the volume fraction of one or more fluids. Within each control volume, the sum of the volume fractions of all the phases is 1. As to Phase $q$, its equation is:

$$
\frac{\partial a_{q}}{\partial t}+\frac{\partial\left(u a_{q}\right)}{\partial x}+\frac{\partial\left(v a_{q}\right)}{\partial y}+\frac{\partial\left(w a_{q}\right)}{\partial z}=0
$$

where $\sum_{q=1}^{2} a_{q}=1 \quad(q=1,2), q=1$ represents the air phase, $q=2$ represents the water phase, $a_{q}$ is the 
volume fraction of the $q$-th phase and $a_{q}=0.5$ is defined as the interface of air and water.

\subsubsection{Multi-degree of freedom module}

For predicting the motions of a ship in waves, two reference coordinate systems are established. One is an earth-fixed system and the other is a moving system. The origin of the moving coordinate system is at the center of mass of the ship. The equations of motions can be written as:

$$
\begin{gathered}
\frac{\mathrm{d} B}{\mathrm{~d} t}+\Omega \times B=F \\
\frac{\mathrm{d} K}{\mathrm{~d} t}+\Omega \times K+V \times B=M
\end{gathered}
$$

Where $B$ is the momentum of the ship, $\Omega$ is the angular velocity, $F$ is the external force, $K$ is the moment of momentum, $V$ is the ship speed and $M$ is the resultant moment.

The dynamic fluid body interaction (DFBI) module is an effective method to simulate realistic ship behaviour in multiple degrees of freedom. In the calculation, the motions of ships are realized by updating the boundary conditions, and not the mesh deformation. At each time-step, the RANS solver is used to calculate the excitation forces and moments acting on the hull surface, and solve the ship motion equations in order to obtain the acceleration, velocity and displacement (Tezdogan et al., 2015). According to the position of the hull and the two-phase flow distribution of the velocity inlet, the free surface position (volume fraction) is updated in order to achieve the movement of grids (Wang, et al., 2014). Then the position of the hull can be changed.

\subsubsection{Time-step selection}

Courant-Friedrichs-Lewy (CFL) implies that a large CFL will decrease precision, whereas a small CFL will increase the convergence time. Therefore, the selection of a suitable time step is of great importance. For the simulation of the ship responses in waves, ITTC (2011b) recommends that a minimum of 100 time steps per encounter period should be used on the CFD model. In the study of Carrica (2007), the forward speed diffraction problem for a DTMB5512 was simulated using $\Delta t=$ 0.00683 as the time step at the medium speed case $(F r=0.28)$ at $\lambda / L_{p p}=1.5$, and $a k=0.025$. As the study in this paper, the time step is nearly the same as the Carrica's paper, that is, $\Delta t=0.005$ was used throughout all simulation cases, resulting in 165 time steps per encounter period.

Because of the influence of waves, CFL number is not a fixed value. To illustrate the CFL number used in this paper, Fig. 1 demonstrates the convective courant number on the hull surface at $t / T=0$. The average value of the convective courant number is 0.157 , which corresponds to the requirement reported by Tezdogan et al. (2015).
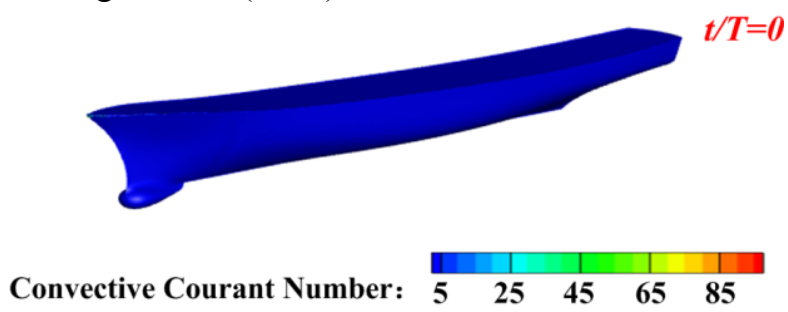

Fig. 1. The convective courant number on the hull surface at $t / T=0$

\subsection{The establishment of a numerical wave tank}

\subsubsection{Wave generation}

The establishment of a numerical wave tank is necessary to calculate the total resistance of a ship in waves. According to the first order wave theory, the surface elevation equation of the incident waves at the velocity inlet boundary can be written as: 


$$
\eta=a \cos \left(k x-\omega_{e} t\right)
$$

The wave number $k$ is given as:

$$
k=2 \pi / \lambda
$$

The encounter frequency $\omega_{\mathrm{e}}$ is defined as:

$$
\omega_{e}=\omega_{0}+k U
$$

Where $a$ is the wave amplitude, $\lambda$ is the wavelength, $U$ is the ship speed, $\omega_{0}$ is the natural frequency, determined by $\omega_{0}=\sqrt{2 \pi g / \lambda}$.

\subsubsection{Numerical wave damping}

The establishment of the damping region is necessary for preventing the influence of the reflected wave. The damping model provided by STAR-CCM+ is used to dampen the waves. The wave dissipation formula (Choi and Sung, 2009) is deduced as follows:

$$
s_{z}^{d}=\rho\left(f_{1}+f_{2}|\beta|\right) \frac{e^{\kappa}-1}{e^{1}-1} \beta
$$

Where $\kappa=\left(\frac{x-x_{s d}}{x_{e d}-x_{s d}}\right)^{n^{d}}, x_{s d}$ is the starting point of the absorbing region, $x_{e d}$ is the outlet boundary of the wave tank, $f_{1}, f_{2}$ and $n_{d}$ are the parameters of the model, and $\beta$ is the vertical velocity component.

\subsection{Computational domains and boundary conditions}

Two different computational domains are built for DTMB5512 and WIGLEY III. In order to improve the calculation efficiency, only half of the hull is used to simulate the hydrodynamic performance. According to the requirement of the overset mesh, the whole model needs two individual blocks which are named as the background block and the overset block. The background block is only a cuboid, and the overset block is a model with Boolean subtraction between the cuboid and the hull. ITTC (2011b) recommends that the inlet boundary should be positioned $1-2 L_{P P}$ away from the hull, and the outlet boundary should be located $3-5 L_{P P}$ downstream to avoid the influence of wave reflection. Therefore, in this paper, for the background block, the length in front, back, top, bottom and left of the hull are taken as $1 L_{p p}, 6 L_{p p}, 1 L_{p p}, 4 L_{p p}$ and $3 L_{p p}$, respectively (where $L_{p p}$ is the length between perpendiculars of the hull). For the overset block, the length in front, back and left of the hull are taken as $0.5 L_{p p}, 1.5 L_{p p}$ and $2 L_{p p}$, respectively. In order to avoid the influence of the wave reflection at the outlet boundary, Qi and Wang (2003) pointed out the wave damping region should be located $1-2 L_{p p}$ in downstream. Therefore, the length of the damping region is set as $1.5 L_{p p}$ away from the outlet boundary on the background region in this paper.

Fig. 2 shows the boundary conditions of the computational domains. For the background block, the top, bottom, and front boundaries are selected as velocity inlets. The purpose of selecting the velocity inlet at the front boundary is to generate regular waves. The aim of using the velocity inlet at the top and bottom boundaries is to prevent the fluid from sticking to the walls, and to represent a deep water and infinite air condition. The back boundary is selected as the pressure outlet in order to stop any backflow and fix the static pressure. Tezdogan et al. (2015) pointed out that the side boundaries can be selected as slip-walls or symmetry planes, because they do not have a great influence on the calculation of the total resistance. Therefore, in this paper, the right side of the tank is selected as a symmetry plane. The left side of the tank is also selected as a symmetry plane to improve the calculation efficiency. For the overset block, the right side of the cuboid is set as a symmetry plane. The rest of the surface is set as overset mesh to facilitate the transfer of the flow field information. The 
hull is set as a no-slip boundary to calculate the ship resistance and the ship motions.

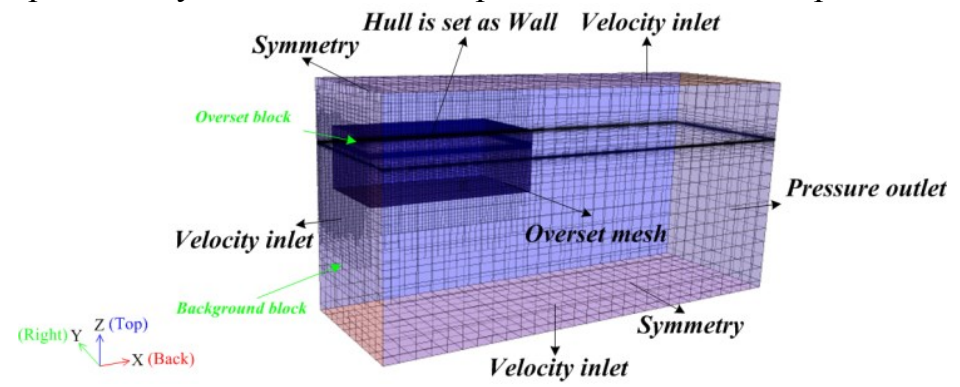

Fig. 2. A general view of the whole computational domain with boundary conditions

\subsection{Mesh generation}

Overset mesh is a new mesh generation scheme with high-quality grids and high-accuracy resistance prediction, and it has been used in many studies in this area (Carrica et al., 2007; Yang et al., 2015; Tezdogan et al., 2015). Therefore, in this paper, an overset mesh is generated for the whole solution domain with linear interpolation. In order to simulate the motions of ships in waves, the cell numbers need to follow the recommendations of ITTC (2011b). According to the requirements, a minimum of 80 cell numbers per wavelength should be used on the free surface and a minimum of 20 cell numbers should be used in the vertical direction. In this study, the computation mesh has been refined in the vertical direction for 40 cell numbers to simulate the regular waves, as shown in Fig. 2. Fig. 3 shows the cross-section on the free surface, and the mesh is clustered near the hull for the sake of capturing the Kelvin wave clearly, and 80 cell numbers per wavelength has been used in the positive $x$-direction with the purpose of capturing the severe free surface flows. Fig. 4 and Fig.5 show the bow mesh and the stern mesh for the DTMB5512 and WIGLEY III. After the completion of the mesh, the cell numbers of these two computational domains total around $3.9 \times 10^{6}$.

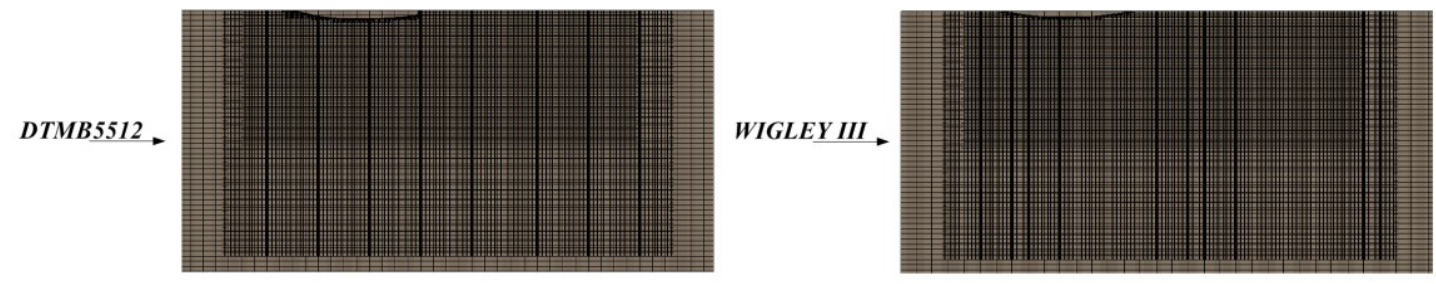

Fig. 3. Mesh on the free surface

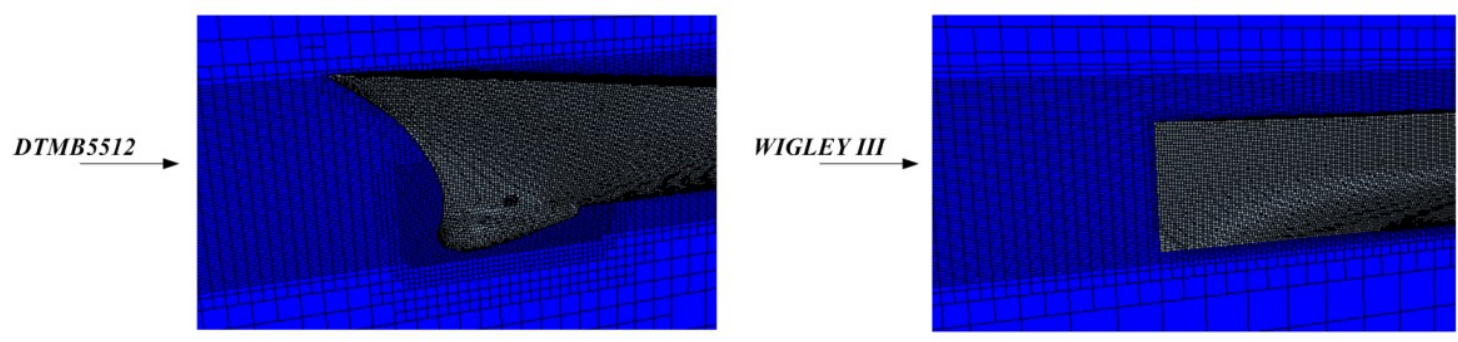

Fig. 4. Bow mesh

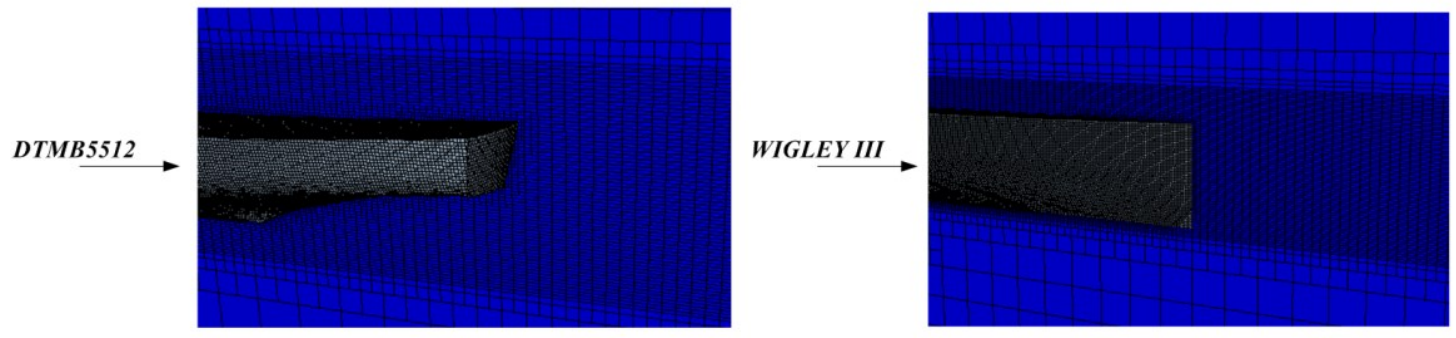

Fig. 5. Stern mesh 


\section{Optimization methodology}

\subsection{Geometry regeneration}

Based on B-spline technique, the arbitrary shape deformation (ASD) technique is an effective method to change the shape of different geometries using the commercial software (Sculptor). It can improve the efficiency of geometric reconstruction with few design variables. In the optimum design, the geometry is modified freely, and the smoothness of new surfaces is good, even under large deformation conditions. This direct deformation method provides the possibility for the optimization of complex geometries (Sun et al., 2010). The specific steps are as follows:

1. The ASD volume is built around the CAD model with the control points and the connections. The deformation volume can be finer or coarser, depending on the shape change control desired.

2. Change the positions and directions of the control points.

3. Obtain the new geometry.

Taking a sphere as an example, first of all, the ASD volume is built around the sphere, as shown in Fig. 6. Secondly, the positions and directions of point 1 and point 2 are changed. Finally, the new geometry is generated.

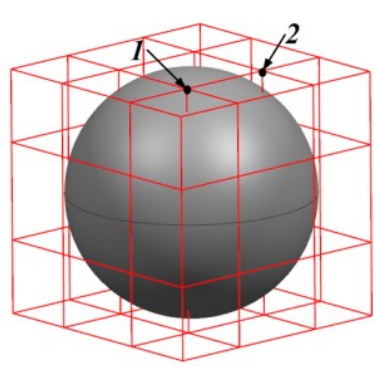

Original sphere

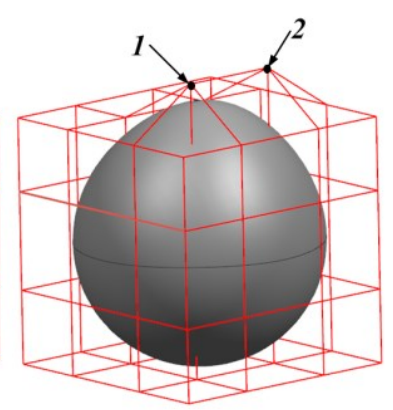

Modified sphere

Fig. 6. Geometry regeneration

\subsection{Hybrid optimization algorithm}

As the weakness of the NLPQL algorithm has already been discussed in the introduction, this section will outline the hybrid optimization algorithm, combining the Opt LHD and the NLPQL algorithm, for solving the hull form optimization problems.

\subsubsection{Optimal Latin hypercube design (Opt LHD)}

There are various forms of design of experiments (DOE) methods, such as central composite, orthogonal design, full factorial design etc. Among them, the random Latin hypercube design algorithm has been improved to obtain better uniformity, space-filling and equilibrium, which is known as the optimal Latin hypercube design (Opt LHD) algorithm. In this, the matrix generating step is as follows:

$m$ test points, $n$ factors constitute the $n * m$ matrix (Morris and Mitchell, 1995):

$$
x=\left[x_{1}, x_{2}, x_{3}, \ldots \ldots, x_{m}\right]
$$

Analyze the $i$-th test point:

$$
x_{i}^{T}=\left[x_{i 1}, x_{i 2}, x_{i 3}, \ldots . ., x_{i n}\right]
$$

The Latin hypercube design algorithm is used to generate an initial design matrix according to the formula (13), and then update the design matrix through element exchange. Finally, optimal space filling is obtained by the principle of max-min distance: 


$$
d\left(x_{i}, x_{j}\right)=d_{i j}=\left[\sum_{k=1}^{n}\left|x_{i k}-x_{j k}\right|^{\theta}\right]^{\frac{1}{\theta}}
$$

Where $\theta=1$ or $2,1 \leq i, j \leq m, i \neq j$ and the sampling point $d\left(x_{i}, x_{j}\right)$ is the minimum distance between $x_{i}$ and $x_{j}$.

Take 3 levels of 3 factors as an example. Fig. 7 shows the sample distributions by random Latin hypercube design (a) and optimal Latin hypercube design (b). It can be seen that the samples are more uniform and accurate, with improved spatial distribution, with the optimal Latin hypercube design (b).

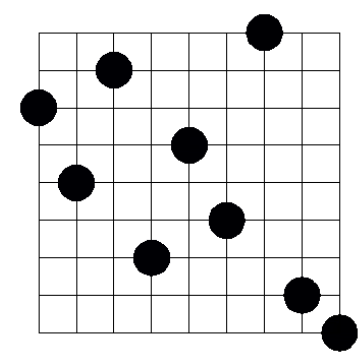

(a) Random Latin Hypercube Design

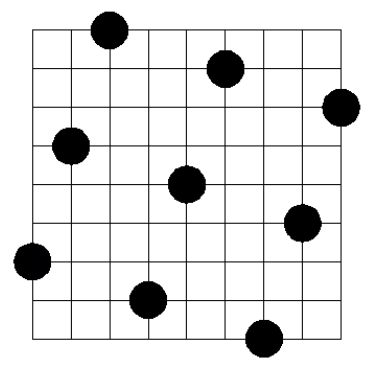

(b) Optimal Latin Hypercube Design

Fig. 7. Comparison of Random Latin Hypercube Design (a) and Optimal Latin Hypercube Design (b)

\subsubsection{Non-linear programming by quadratic Lagrangian (NLPQL)}

Non-linear programming by quadratic Lagrangian (NLPQL) (Schittkowski, 1985) is used as the optimization method to find the optimal hull form. This method is the modified SQP algorithm and it has the advantages of high-stability and fast convergence. The objective function is expanded by Taylor Series by linearizing the non-linear constraints and the next design point can be obtained by solving the quadratic programming. Then, a linear search is performed according to two alternative optimization functions. The flow chart for the NLPQL method has been list in the Fig. 8. First, beginning with an initial guess point $X_{0}$, an additional linear search is applied in the calculation process to ensure that it is able to reach the global convergence. $X_{k+1}=X_{k}+a_{k} d_{k}$ will be carried out using the new iteration only when $X_{k+1}$ as a feasible search plan confirms the moving step length along this direction. The Broyden-Fletcher-Goldfarb-Shanno (BFGS) algorithm is adopted in NLPQL and a variable scale matrix $B_{k}$ constructs $B_{k+1}$ to approach the Hessian matrix so as to complete the updating of $B_{k}$. This non-linear programming problem can be described as follows:

The objective function:

$$
q_{k}(d)=\frac{1}{2} d^{T} B_{k} d+\nabla f\left(x_{k}\right)^{T} d \quad, d \in R^{n}
$$

The constraint equations:

$$
\begin{gathered}
\nabla g_{i}\left(x_{k}\right)^{T} d+g_{i}\left(x_{k}\right) ; i=1,2, \ldots \\
\nabla h_{j}\left(x_{k}\right)^{T} d+h_{j}\left(x_{k}\right)=0 ; j=1,2, \ldots
\end{gathered}
$$




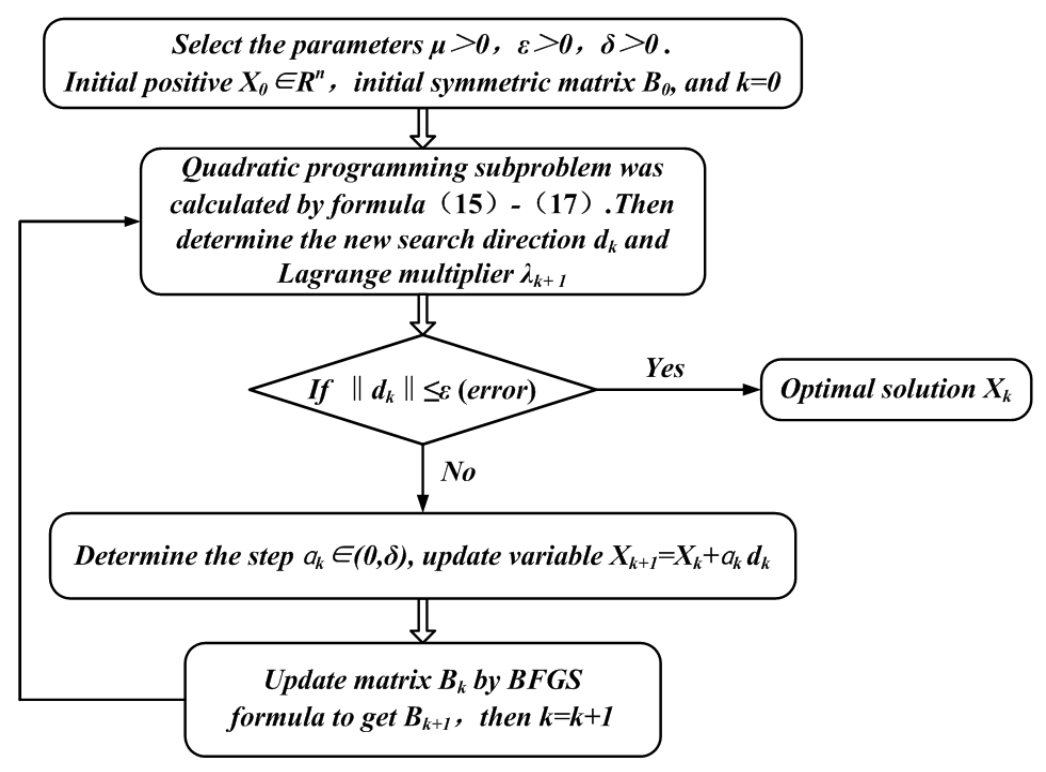

Fig. 8. Flow chart for the NLPQL method

\subsubsection{Optimization loop}

In this paper, a hull form optimization loop is developed, and the general flow chart is given in Fig. 9. An overview of the processes depicted in Fig. 9 is given in the following steps:

1. Opt LHD is used to sample the design space, and the solution of minimum total resistance is set as the initial point of the NLPQL.

2. Use ASD technique to change the geometry.

3. Generate the new hull form and computational domain.

4. Calculate the displacement of this new hull form, and then compare with the displacement constraint. If the displacement of the new hull form does not meet the requirement, eliminate this new hull form and repeat Step 2 until the constraint is met.

5. Mesh the computational domain using the overset mesh technique.

6. Calculate the total resistance of the hull in waves using the RANS method.

7. Repeat Steps 2-6 until no further iterations occur. Then output the optimal hull form. 


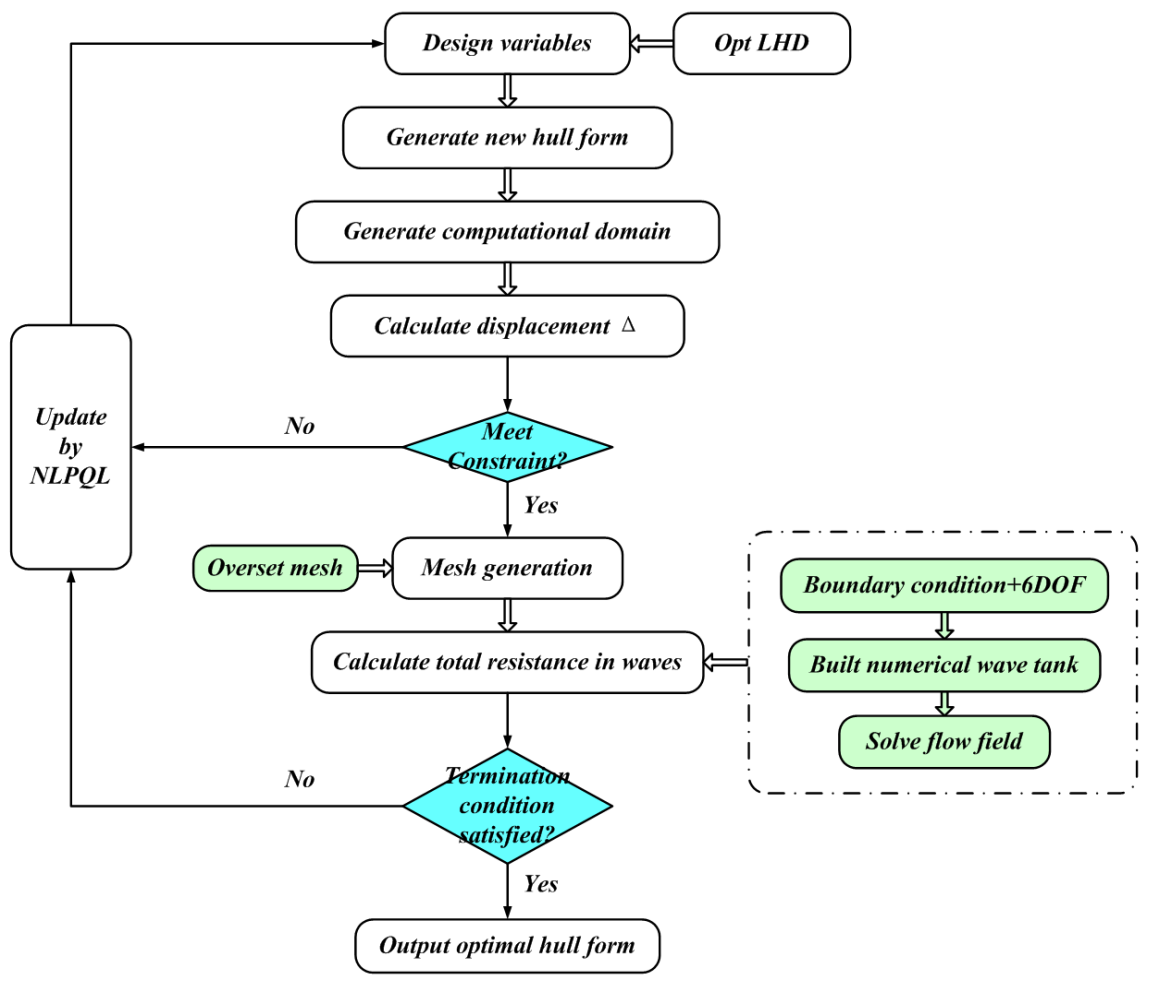

Fig. 9. A hull form optimization loop developed in this study

\section{Results}

\subsection{Post-processing formulations}

\subsubsection{Total resistance coefficient}

For the cases below, the dimensionless total resistance coefficient $C_{t w}$ is used to analyse the total resistance of a ship in waves, and $C_{t w}$ can be obtained by:

$$
C_{t w}=\frac{R_{t w}}{0.5 \rho U^{2} S}
$$

Where $R_{t w}$ is the total resistance of a ship in waves, $\rho$ is the fluid density, $U$ is the speed of a ship, and $S$ is the wetted surface area.

\subsubsection{Motion responses}

As the motions and the forces exhibit a cyclical character due to the form of regular incident waves, Fourier series are employed to analyse the unsteady time histories. Each unsteady history $\varphi(t)$ can be calculated by:

$$
\varphi(t)=\frac{\varphi_{0}}{2}+\sum_{n=1}^{N} \varphi_{n} \cos \left(\omega_{e} n t+\gamma_{n}\right), \quad n=1,2,3, \ldots
$$

Where $\varphi_{n}$ is the n-th harmonic amplitude, and $\gamma_{n}$ is the corresponding phase. These values can be found using the following formulae:

$$
\varphi_{n}=\sqrt{a_{n}^{2}+b_{n}^{2}}
$$


In which:

$$
\gamma_{n}=\tan ^{-1}\left(\frac{b_{n}}{a_{n}}\right)
$$

$$
\begin{aligned}
& a_{n}=\frac{2}{T} \int_{0}^{T} \varphi(t) \cos \left(\omega_{e} n t\right) d t \\
& b_{n}=-\frac{2}{T} \int_{0}^{T} \varphi(t) \sin \left(\omega_{e} n t\right) d t
\end{aligned}
$$

In order to make a quantitative analysis on ship motions, the transfer functions of the heave and pitch can be defined as:

$$
\begin{aligned}
& T F_{3}=\frac{x_{3_{1}}}{a} \\
& T F_{5}=\frac{x_{5_{1}}}{a k}
\end{aligned}
$$

Where $T F_{3}$ and $T F_{5}$ are the transfer functions of the heave and pitch motions, respectively, $x_{31}$ is the first Fourier series harmonic amplitude of heave, and $x_{5_{1}}$ is the first Fourier series harmonic amplitude of pitch.

\subsection{Hull A-DTMB5512 bow optimization}

DTMB5512 was initially designed as a USA Navy surface combatant. This model has a sonar dome and transom stern with scale of 1:46.6. Table 1 shows the geometrical properties of DTMB5512. Table 2 shows the wave conditions which are investigated in this paper, and Case 2 in Table 2 is selected for the optimization.

Table 1 Geometrical properties of the DTMB5512

\begin{tabular}{cc}
\hline Main particulars & Value \\
\hline Scale factor & $1: 46.6$ \\
Length $L_{p p}(\mathrm{~m})$ & 3.048 \\
Breadth $B(\mathrm{~m})$ & 0.409 \\
Draft $D(\mathrm{~m})$ & 0.132 \\
Displacement $\Delta(\mathrm{kg})$ & 86.4 \\
Block Coefficient $C_{B}$ & 0.507 \\
Wetted surface area $S\left(\mathrm{~m}^{2}\right)$ & 1.371 \\
Vertical center of gravity (from keel) $K G(\mathrm{~m})$ & 0.162 \\
Moment of Inertia $K_{y y} / L_{p p}$ & 0.25 \\
\hline
\end{tabular}

Table 2 Wave conditions

\begin{tabular}{cccccc}
\hline Case $N o$. & $F r$ & Wave steepness $a k$ & $\lambda / L_{p p}$ & Amplitude $a(\mathrm{~m})$ & Encounter freq. $f_{\mathrm{e}}(\mathrm{Hz})$ \\
\hline 1 & 0.19 & 0.025 & 1 & 0.0121 & 1.0562 \\
$\mathbf{2}$ & $\mathbf{0 . 2 8}$ & $\mathbf{0 . 0 2 5}$ & $\mathbf{1}$ & $\mathbf{0 . 0 1 2 1}$ & $\mathbf{1 . 2 1 7 6}$ \\
3 & 0.34 & 0.025 & 1 & 0.0121 & 1.3251 \\
\hline
\end{tabular}

\subsubsection{Grid sensitivity study}

For the mesh convergence study, Tezdogan et al. (2015) pointed out that the mesh needs to be changed only on the overset region, and the background region should not alter. Because the fine mesh configurations on the background region is necessary for capturing the wave accurately. Therefore, in order to study the grid sensitivity, the mesh is altered only on the overset region with the grid refinement ratio $r=\sqrt{2}$, as successfully applied by Tezdogan et al. (2015) and Kavli et al. (2017). 
Table 3 shows the results of the total resistance coefficient $C_{t w l}$ changes with the different mesh configurations at $F r=0.28$ in Case 2 . The total resistance coefficient is over predicted using the coarse mesh and medium mesh, and is under predicted using the fine mesh. The variation in total resistance coefficient is below $3.1 \%$, and the result obtained by fine mesh is closer to the experimental value. Therefore, the fine mesh has been used in the hull form optimization in this study.

Table 3 Total resistance coefficient $C_{t w l}$ calculated at different mesh configurations at Case 2

\begin{tabular}{cccc}
\hline Mesh configurations & Total cell numbers & $C_{t w 1}$ & Error (\%) \\
\hline Coarse & 1146281 & 0.004947 & 5.12 \\
Medium & 1829810 & 0.004689 & 2.46 \\
Fine & 3829007 & 0.004574 & -0.56 \\
\hline
\end{tabular}

\subsubsection{Optimization strategy}

Fig. 10 shows the optimization problem of DTMB5512 case. Design variables, control parameters and constraints are provided in Table 4.

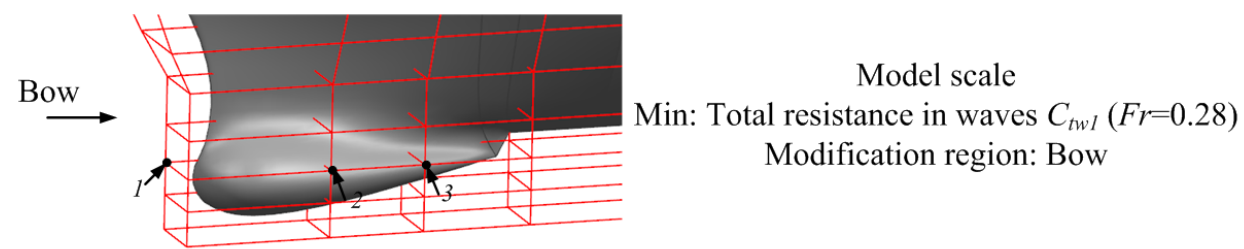

Fig. 10. Problem definition of the optimal design of the DTMB5512 case

Table 4 Parameters for the DTMB5512 model

\begin{tabular}{|c|c|c|c|c|}
\hline & \multicolumn{4}{|c|}{ Design variables } \\
\hline Parameters & $a_{11}$ & $a_{12}$ & $a_{21}$ & $a_{22}$ \\
\hline No. & 1 & 2 & 3 & 1 \\
\hline Movement Direction & $x$ & $y$ & $y$ & $z$ \\
\hline Constraints & $-0.4 \leq a_{11} \leq 0$ & $-0.15 \leq a_{12} \leq 0.02$ & $-0.15 \leq a_{21} \leq 0.1$ & $-0.13 \leq a_{22} \leq 0.1$ \\
\hline \multirow{3}{*}{ Displacement constraint } & & $\Delta_{n e w}-\Delta_{o r g}$ & \multirow{2}{*}{$\leq 0.01$} & \\
\hline & & $\Delta_{\text {org }}$ & & \\
\hline & Where & the original hull, & new means the mc & fied hull. \\
\hline
\end{tabular}

\subsubsection{Optimization and numerical results analysis for DTMB5512 ship}

As explained above, the first step of optimization is the design of experiments (DOE) for the hybrid optimization algorithm. DOE can explore the influence of the four parameters effectively. After the DOE, a set of design variables with minimum total resistance coefficients can be selected as the initial point of the NLPQL algorithm. This step can improve the optimization accuracy of the NLPQL algorithm which has been verified below. Table 5 shows the DOE numerical results by using Opt LHD method. Fig. 11 shows the space distributions of samples. 
Table 5 Samples by Opt LHD

\begin{tabular}{cccccc}
\hline No. & $a_{11}$ & $a_{12}$ & $a_{21}$ & $a_{22}$ & $C_{t w 1}$ \\
\hline 1 & -0.0201 & -0.02442 & 0.0623 & -0.0479 & 0.004599 \\
2 & -0.2533 & -0.03809 & 0.0573 & -0.0468 & 0.004543 \\
3 & -0.1065 & -0.09362 & -0.1148 & 0.0884 & 0.004564 \\
4 & -0.201 & -0.02528 & -0.0143 & -0.0214 & 0.004503 \\
5 & -0.3075 & -0.08337 & -0.0721 & -0.1115 & 0.004651 \\
6 & -0.2432 & -0.01503 & -0.145 & -0.019 & 0.004509 \\
7 & -0.3176 & -0.0808 & -0.0759 & -0.0329 & 0.004554 \\
8 & -0.0422 & -0.00392 & -0.1136 & 0.0769 & 0.004626 \\
9 & -0.0683 & -0.10131 & -0.0683 & -0.0075 & 0.004643 \\
10 & -0.203 & -0.13975 & 0.0824 & 0.0561 & 0.004658 \\
$\ldots$ & $\ldots$ & $\ldots$ & $\ldots$ & $\ldots$ & $\ldots$ \\
$\ldots$ & $\ldots$ & $\ldots$ & $\ldots$ & $\ldots$ & $\ldots$ \\
197 & -0.3437 & -0.06286 & 0.0673 & 0.0792 & 0.004563 \\
198 & -0.3276 & -0.07739 & 0.0523 & 0.0098 & 0.004564 \\
199 & -0.2854 & -0.10387 & -0.0043 & 0.033 & 0.00453 \\
200 & -0.0121 & -0.08764 & 0.0611 & 0.0353 & 0.004685 \\
\hline
\end{tabular}
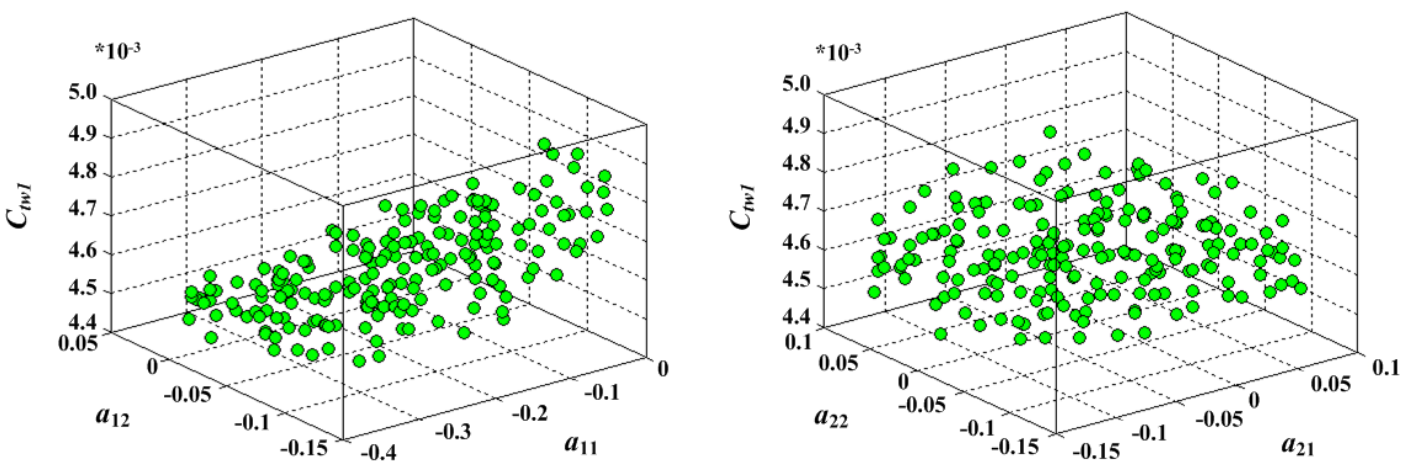

Fig. 11. Samples of the DTMB5512 case

The optimization loop was carried out on the ARCHIE-WeST High Performance Computer (http://www.archie-west.ac.uk) and each generation was computed for approximately 72 CPU hours. After the optimization, an optimal hull form (optimized hull-A) has been found, as shown in Table 6 . For the optimized hull-A, the total resistance decreases by $3.71 \%$. Although the shape of the bow is changed, there are insignificant differences for $T F_{3}$ and $T F_{5}$ between the original hull and the optimized hull-A. Fig. 12 shows a comparison of the bow sections for the original hull and the optimized hull-A.

Table 6 Optimization results

\begin{tabular}{cccccc}
\hline \multirow{2}{*}{ Name } & $F r$ & $\frac{C_{t w 1-o r g}}{C_{t w 1-o p t}}$ & $\frac{\Delta_{\text {org }}}{\Delta_{\text {opt }}}$ & $\frac{T F_{3-o r g}}{T F_{3-o p t}}$ & $\frac{T F_{5-o r g}}{T F_{5-o p t}}$ \\
\hline Optimized Hull-A & 0.28 & 1.0385 & 1.0069 & 0.9918 & 1.0361 \\
\hline
\end{tabular}

In Table 6: opt means the optimal hull. 

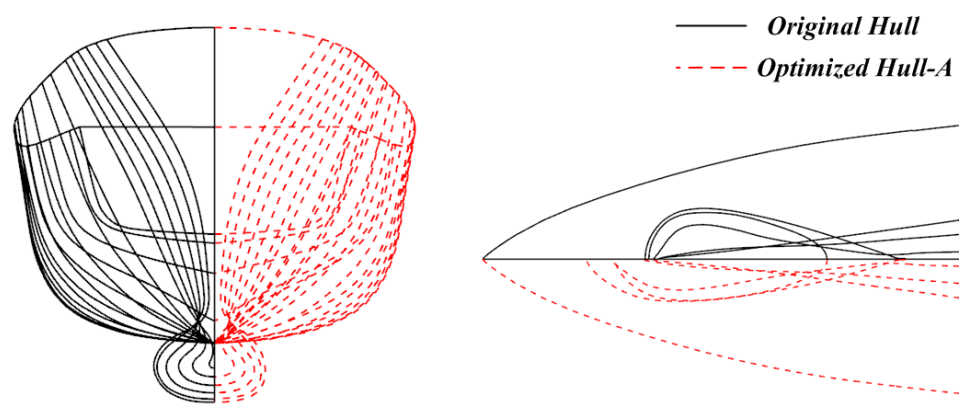

Fig. 12. Comparison of geometry for the original hull and the optimized hull-A

Fig. 13 shows a comparison of the evolution history of $C_{t w 1}$, and each point represents the total resistance of the different hull forms obtained through optimization. It can be seen that the total resistance of the best ship decreases by $3.71 \%$ at 8 iterations and $2.55 \%$ at 12 iterations for the hybrid algorithm and the NLPQL algorithm, respectively. The numerical test showed that different initial points gave different optimal results, and the drag reduction effect is better using the hybrid algorithm. Since the optimization is only carried out at $F r=0.28$, further calculations were performed to predict the ship behavior and performance at different $F r$ values at $\lambda / L_{p p}=1, a k=0.025$, and a comparison is made against the original hull and experimental fluid dynamics (EFD) (Gui et al., 2001; Gui et al., 2002; Irvine et al., 2008), as shown in Fig. 14. The model in this paper under predicted the total resistance coefficients, and the deviations between the CFD and EFD data are in the range of $0.5 \%$ to $4 \%$. It can be found that numerical results are in good agreement with the experiment results and the average error between the CFD data and the EFD data is $2.41 \%$. Accordingly, the current CFD model can be used for the calculation of the total resistance which lays the foundation for the ship hull form optimization. For the optimized hull-A, it has a satisfactory resistance reduction effectiveness at different $F r$, and the resistance reduction is greater at $F r=0.34$. Table 7 shows the comparison of the total resistance coefficients per unit displacement. Fig. 15 and Fig. 16 show the $T F_{3}$ and $T F_{5}$ for the original hull and the optimized hull-A at different $F r$ at $\lambda / L_{p p}=1, a k=0.025$.

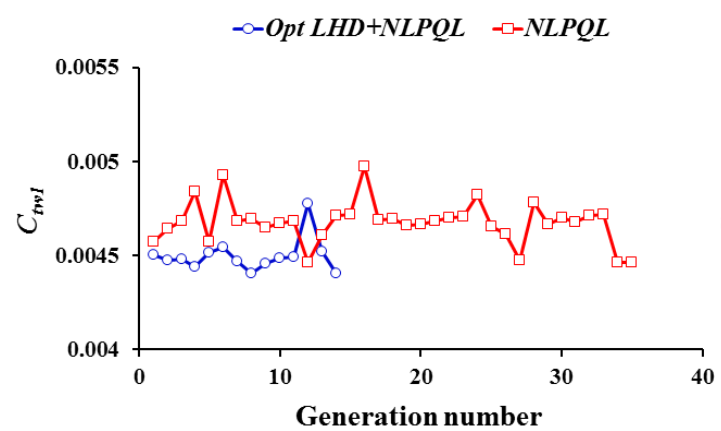

Fig. 13. Evolution history of $C_{t w l}$

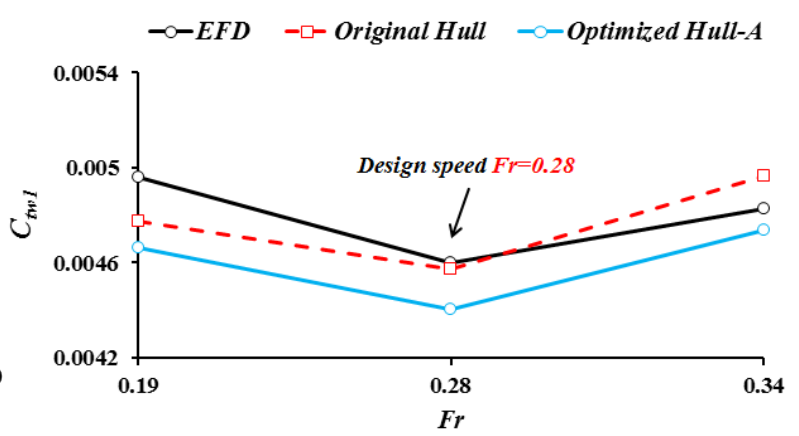

Fig. 14. $C_{t w l}$ changes with different $F r$

Table 7 Resistance comparisons per unit displacement

\begin{tabular}{cccc}
\hline$F r$ & Original Hull & Optimized Hull-A & Reduction\% \\
\hline 0.19 & $5.523 * 10^{-5}$ & $5.437^{*} 10^{-5}$ & 1.56 \\
0.28 & $5.294 * 10^{-5}$ & $5.132^{*} 10^{-5}$ & 3.04 \\
0.34 & $5.747 * 10^{-5}$ & $5.523 * 10^{-5}$ & 3.89 \\
\hline
\end{tabular}




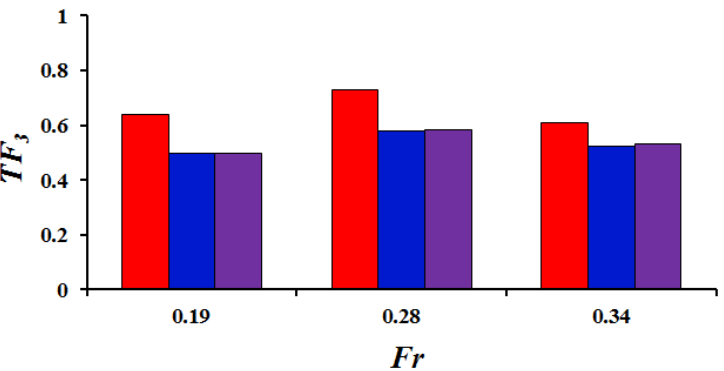

Fig. 15. $T F_{3}$ changes with different $\mathrm{Fr}$

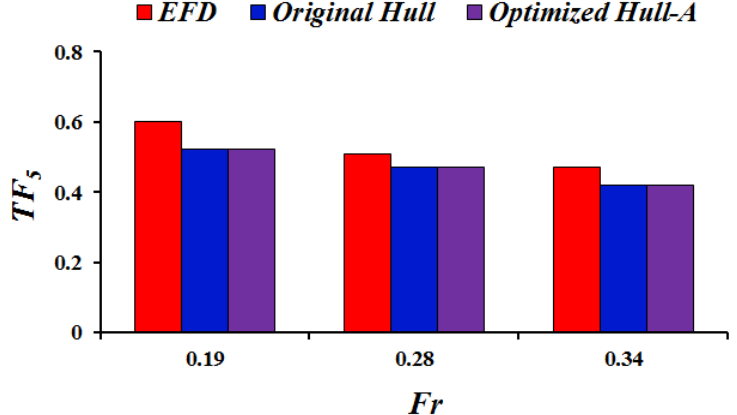

Fig. 16. $T F_{5}$ changes with different $F r$

Fig. 17 shows the comparison of the static pressure between the original hull and the optimized hull-A in an encounter period. The two ships have the smallest fore draft at $t / T=0.25$ and the biggest fore draft at $t / T=0.75$, which is the same as the actual situation.

Fig. 18 shows the wall shear stress on the bow surface of both the original hull and the optimized hull-A in an encounter period. As shown in the figure, the change in the ship's bow shape has affected the pressure distribution on the bow surface. In the case of the optimized hull-A, the pressure has been decreased significantly, which results in the decrease of the total resistance.

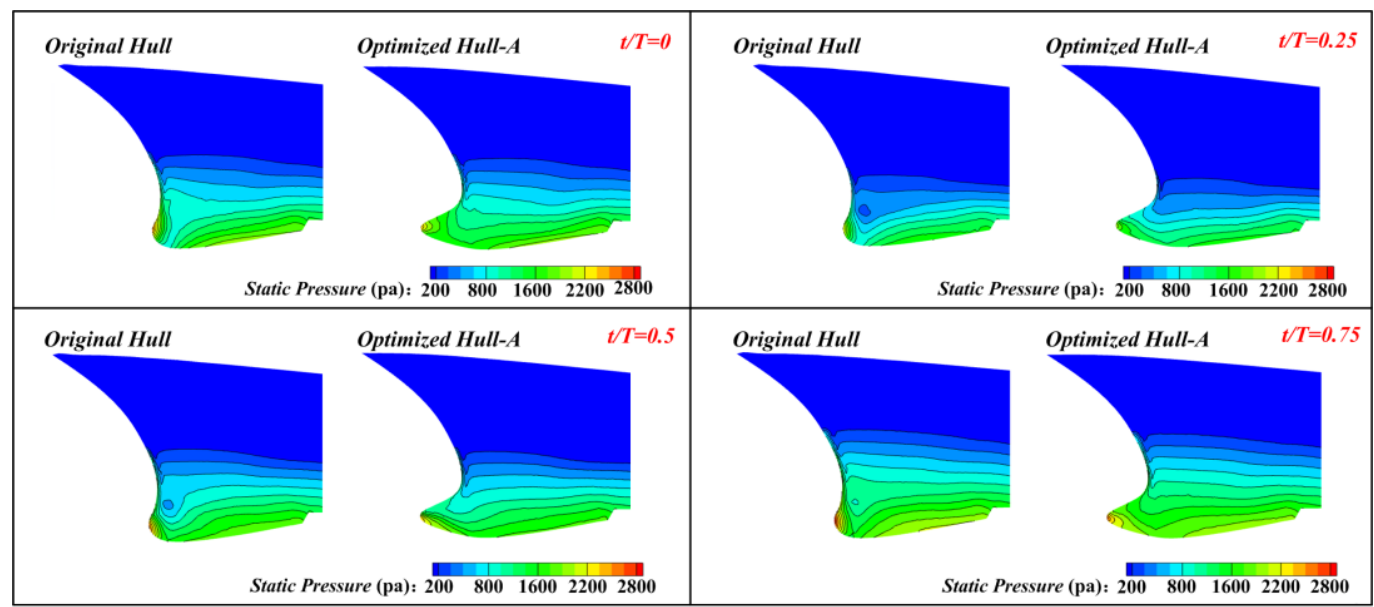

Fig. 17. Comparison of surface pressure in an encounter period

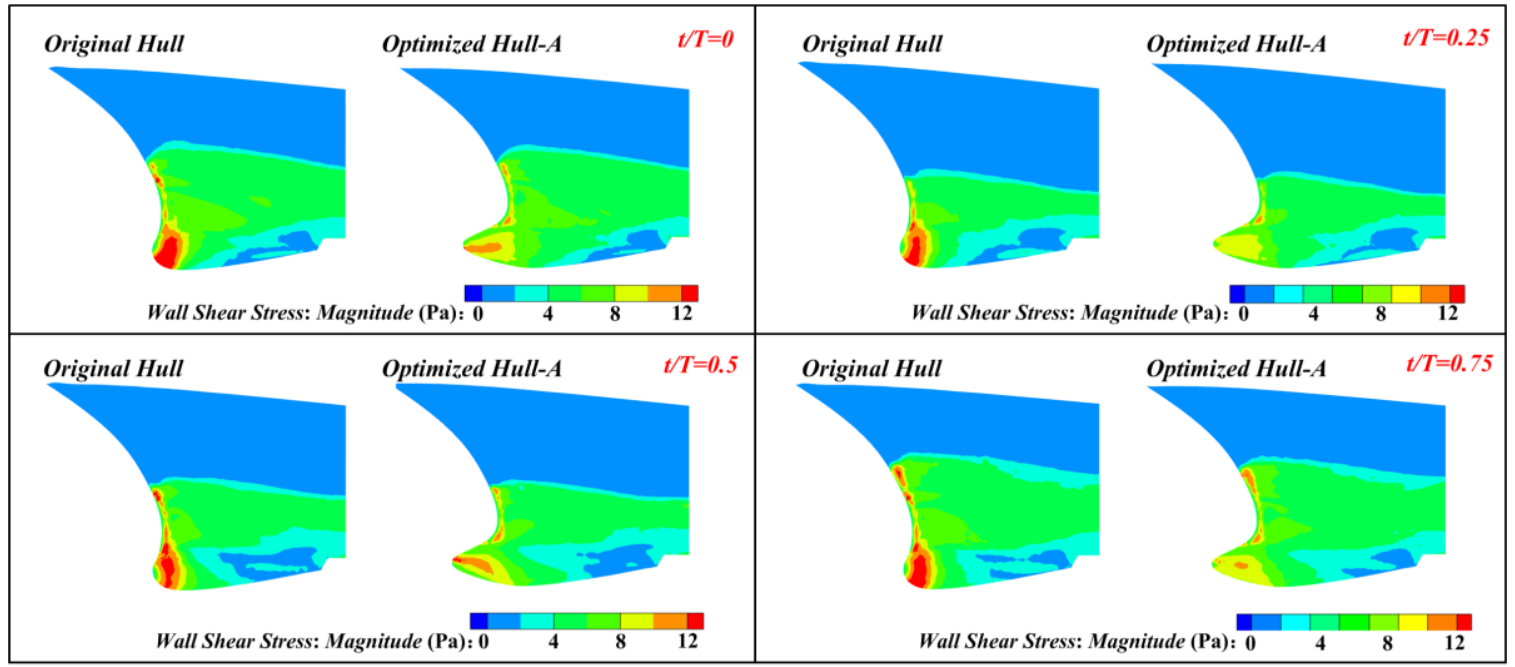

Fig. 18. Contours of the wall shear stress on the bow surface in an encounter period 
Fig. 19 shows the comparison of wave contours for the original hull and the optimized hull-A in an encounter period. It can be seen that significant differences of wave contours are seen near the bow at $t / T=0.25, t / T=0.5$, and $t / T=0.75$, while insignificant differences can be found at $t / T=0$. Therefore, the detail of the flow field near the bow at $t / T=0$ has also been shown in Fig. 19. As can be seen in the figure, the new bow shape of optimized hull-A has reduced the bow waves and shoulder waves which results in the reduction of the total resistance.

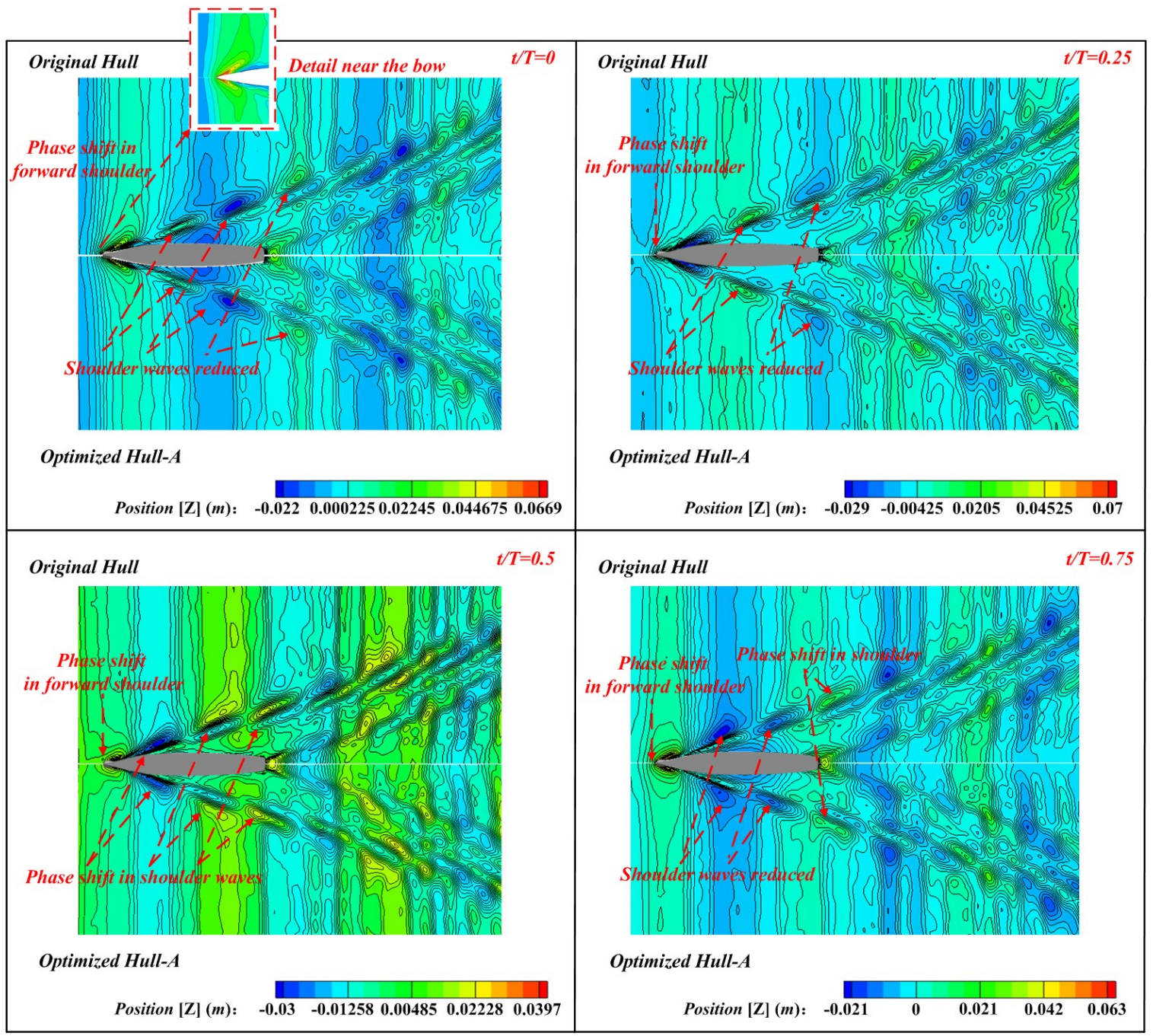

Fig. 19. Details of the free surface wave contours in an encounter period

\subsection{Hull B-WIGLEY III bow optimization}

WIGLEY III is a mathematical hull with a vertical bow and stern which is used among researchers worldwide. For this ship type, Delft University of Technology has performed extensive research on WIGLEY I to WIGLEY IV. The EFD data used in this paper also come from this university (J.M.J.JOURNÉE, 1992). In this paper, WIGLEY III is used as the study object. The ship type can be built using the following formula:

$$
y=\frac{B}{2}\left(1-\left(\frac{z}{D}\right)^{2}\right)\left(1-\left(\frac{2 x}{L}\right)^{2}\right)\left(1+0.2\left(\frac{2 x}{L}\right)^{2}\right)
$$

Table 8 shows the principal dimensions of this model. Table 9 shows the wave conditions which are studied in this paper, and Case 5 is used for the optimization. 
Table 8 Principal dimensions of WIGLEY III

\begin{tabular}{cc}
\hline Main particulars & Value \\
\hline Length $L(\mathrm{~m})$ & 3.00 \\
Breadth $B(\mathrm{~m})$ & 0.30 \\
Draft $D(\mathrm{~m})$ & 0.1875 \\
Displacement $\Delta(\mathrm{kg})$ & 78 \\
Block Coefficient $C_{B}$ & 0.4622 \\
Mertical center of gravity (from keel) $K G(\mathrm{~m})$ & 0.17 \\
Moment of Inertia $K_{y y} / L_{p p}$ & 0.25 \\
\hline
\end{tabular}

Table 9 Wave conditions

\begin{tabular}{cccccc}
\hline Case $N o$. & $F r$ & Wave steepness $a k$ & $\lambda / L$ & Amplitude $a(\mathrm{~m})$ & Encounter freq. $f_{\mathrm{e}}(\mathrm{Hz})$ \\
\hline 4 & 0.2 & 0.023 & 1 & 0.011 & 1.0827 \\
$\mathbf{5}$ & $\mathbf{0 . 3}$ & $\mathbf{0 . 0 2 3}$ & $\mathbf{1}$ & $\mathbf{0 . 0 1 1}$ & $\mathbf{1 . 2 6 3 6}$ \\
6 & 0.4 & 0.023 & 1 & 0.011 & 1.4442 \\
\hline
\end{tabular}

\subsubsection{Optimization strategy}

The optimization problem of the WIGLEY III has been defined in Fig. 20. Design variables, control parameters and constraints are shown in Table 10.

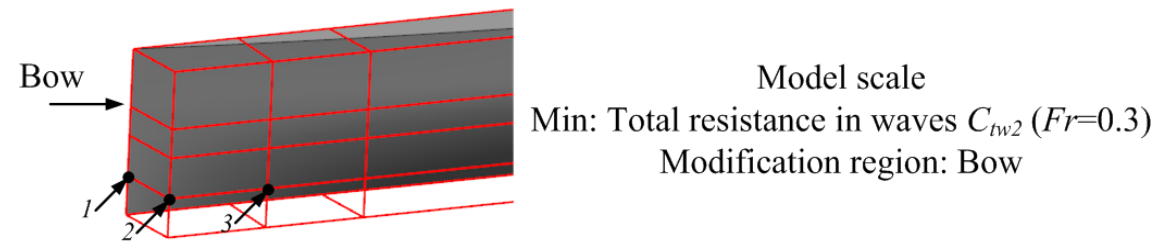

Fig. 20. Problem definition of the optimal design of the WIGLEY III case

Table 10 Parameters for WIGLEY III

\begin{tabular}{c|cccc}
\hline & \multicolumn{4}{|c}{ Design variables } \\
\hline Parameters & $b_{11}$ & $b_{12}$ & $b_{21}$ & $b_{22}$ \\
No. & 1 & 2 & 3 & 1 \\
Movement Direction & $x$ & $y$ & $y$ & $z$ \\
Constraints & $-0.4 \leq b_{11} \leq 0$ & $-0.8 \leq b_{12} \leq 0$ & $-0.8 \leq b_{21} \leq 0$ & $-0.012 \leq b_{22} \leq 0.06$ \\
\hline
\end{tabular}

\subsubsection{Optimization and numerical results analysis for WIGLEY III ship}

Table 11 shows the total resistance of the different hull forms obtained by the Opt LHD method. Fig. 21 shows the space distributions of samples. 
Table 11 Samples by Opt LHD method

\begin{tabular}{c|cccc|c}
\hline No. & $b_{11}$ & $b_{12}$ & $b_{21}$ & $b_{22}$ & $C_{t w 2}$ \\
\hline 1 & -0.0369 & -0.061 & -0.5494 & -0.00275 & 0.005321 \\
2 & -0.3791 & -0.6747 & -0.5237 & 0.04178 & 0.005138 \\
3 & -0.0932 & -0.0161 & -0.7357 & 0.00361 & 0.005363 \\
4 & -0.3068 & -0.2924 & -0.2731 & 0.03976 & 0.005150 \\
5 & -0.249 & -0.7229 & -0.0867 & 0.04294 & 0.005174 \\
6 & -0.008 & -0.1221 & -0.3373 & 0.036 & 0.005252 \\
7 & -0.3936 & -0.1896 & -0.3502 & 0.02993 & 0.005132 \\
8 & -0.1044 & -0.1542 & -0.5783 & 0.01287 & 0.005265 \\
9 & -0.2506 & -0.4305 & -0.1382 & -0.00969 & 0.005189 \\
10 & -0.0369 & -0.061 & -0.5494 & -0.00275 & 0.005174 \\
$\ldots$ & $\ldots$ & $\ldots$ & $\ldots$ & $\ldots$ & $\ldots$ \\
$\ldots$ & $\ldots$ & $\ldots$ & $\ldots$ & $\ldots$ & $\ldots$ \\
197 & -0.2426 & -0.7357 & -0.0964 & 0.00477 & 0.005225 \\
198 & -0.2795 & -0.6683 & -0.0225 & 0.02414 & 0.005140 \\
199 & -0.3598 & -0.1124 & -0.5558 & 0.03022 & 0.005151 \\
200 & -0.2153 & -0.71 & -0.5398 & -0.00448 & 0.005311 \\
\hline
\end{tabular}
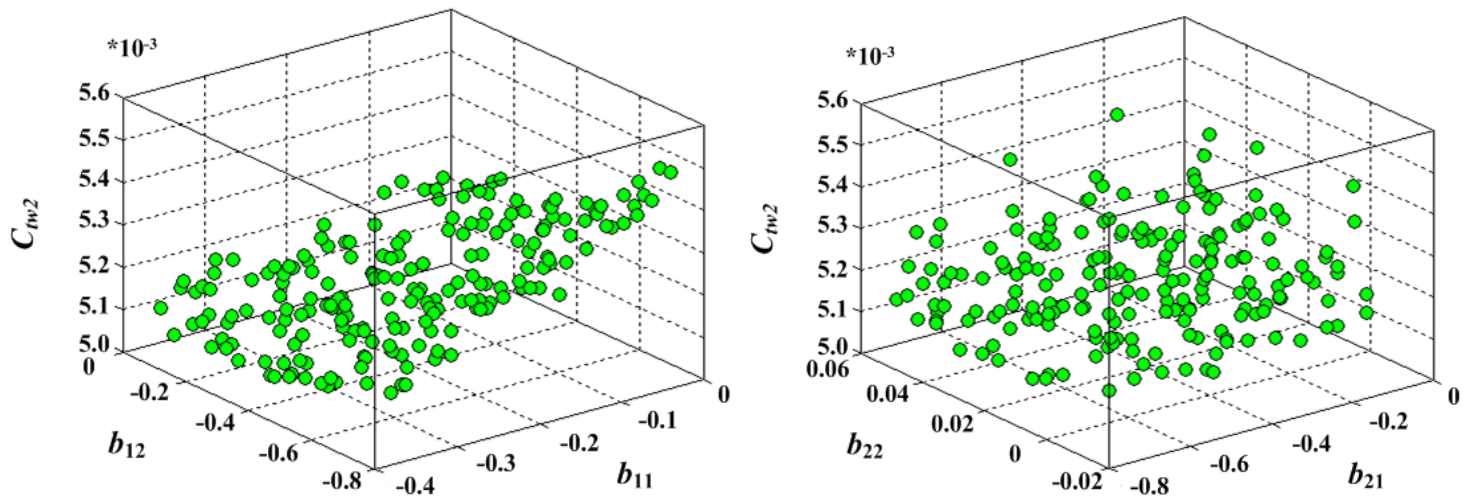

Fig. 21. Samples of WIGLEY III case

Table 12 shows the optimization results. It can be seen from the table that the total resistance decreases by $4.41 \%$ which also signifies the effect of the new bow towards the reduction in resistance. Due to the optimized bow shape, significant differences have been seen for $T F_{3}$ and $T F_{5}$ between the original hull and optimized hull-B. Fig. 22 shows a comparison of bow sections for the original hull and the optimized hull-B. It can be seen that the hull lines of the optimized hull-B are smooth which indicates the validity of the ASD method for changing the geometry.
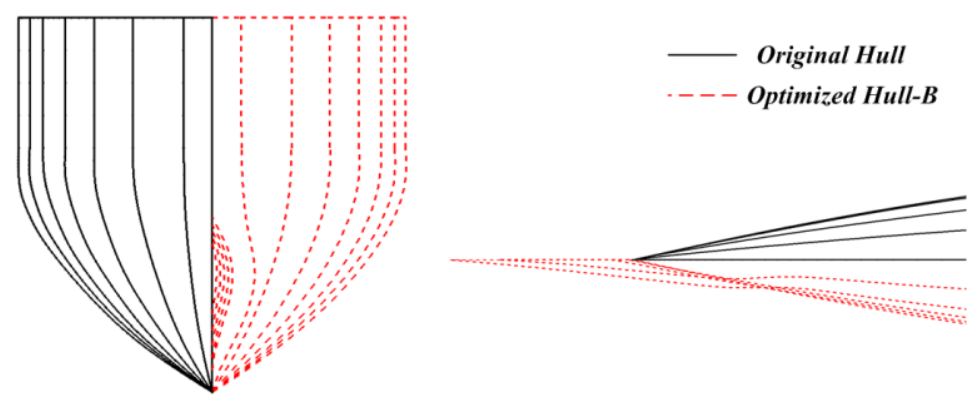

Fig. 22. Comparison of geometry for original hull and the optimized hull-B

Since the new bow of the optimized hull-B changes the displacement of the ship, the resistance 
comparison per unit of displacement has been carried out and details are provided in Table 13. From the table, it can be seen that the total resistance coefficient decreases by $4.94 \%$ at $F r=0.3$, and the effect of a new bow for reduction in resistance coefficient is even greater when $F r=0.2$, which can also be observed from Fig. 23. From Fig. 23, the present CFD model in this paper under predicted the total resistance coefficients within about $7.97 \%$ of the EFD data. The results presented are in good accordance with the EFD data by J.M.J.JOURNÉE (1992). Fig. 24 presents the convergence history between the hybrid algorithm and the NLPQL algorithm. It can clearly be seen that the resistance reduction effect is better with the hybrid algorithm than the NLPQL algorithm. Fig. 25 and Fig. 26 present $T F_{3}$ and $T F_{5}$ for the original hull and the optimized hull-B at different values of $F r$ at $\lambda / L_{p p}=1$, $a k=0.023$.

Table 12 Optimization results

\begin{tabular}{cccccc}
\hline \multirow{2}{*}{ Name } & $F r$ & $\frac{C_{t w 2-o r g}}{C_{\text {tw2-opt }}}$ & $\frac{\Delta_{\text {org }}}{\Delta_{\text {opt }}}$ & $\frac{T F_{3-o r g}}{T F_{3-o p t}}$ & $\frac{T F_{5-o r g}}{T F_{5-o p t}}$ \\
\hline Optimized Hull-B & 0.3 & 1.0461 & 0.9944 & 0.9979 & 1.0387 \\
\hline
\end{tabular}

Table 13 Resistance comparisons per unit displacement

\begin{tabular}{cccc}
\hline Fr & Original Hull & Optimized Hull-B & Reduction\% \\
\hline 0.2 & $6.284 * 10^{-5}$ & $5.916^{*} 10^{-5}$ & 5.85 \\
0.3 & $6.772 * 10^{-5}$ & $6.437 * 10^{-5}$ & 4.94 \\
0.4 & $7.280 * 10^{-5}$ & $6.907 * 10^{-5}$ & 5.12 \\
\hline
\end{tabular}

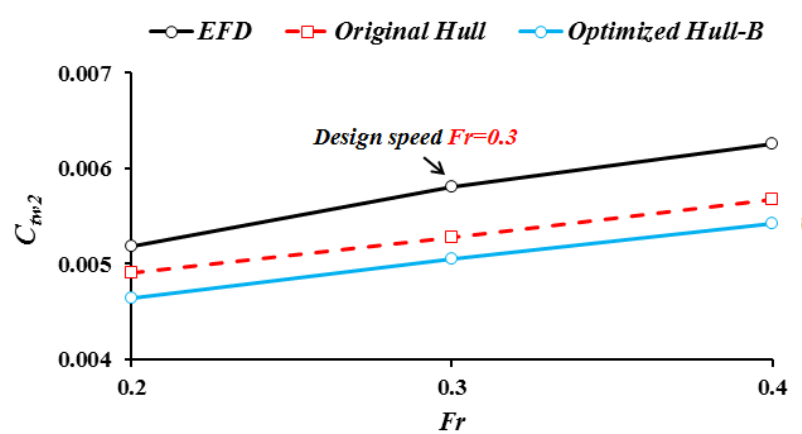

Fig. 23. $C_{t w 2}$ changes with different $F r$

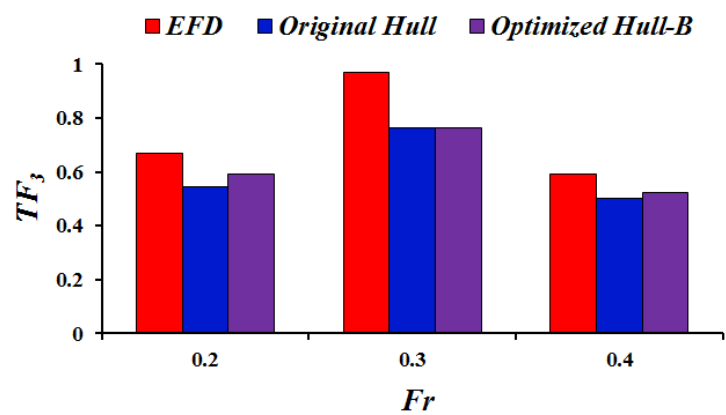

Fig. 25. $T F_{3}$ changes with $\mathrm{Fr}$

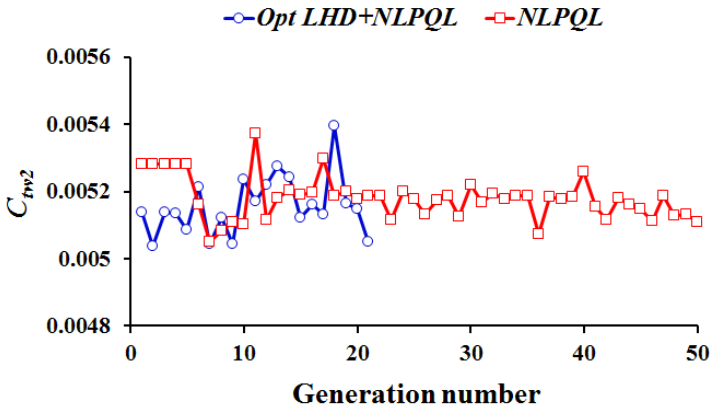

Fig. 24. Evolution history of $C_{t w 2}$

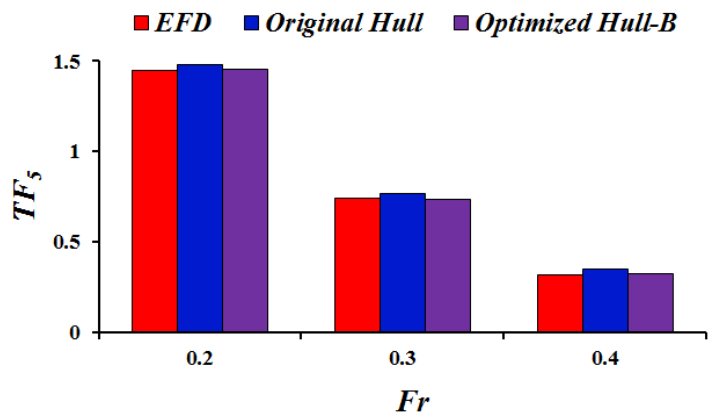

Fig. 26. $T F_{5}$ changes with $\mathrm{Fr}$

Since only the bow shape changed, the detail of the static pressure at the bow has been demonstrated in Fig. 27. The ship keeps minimum bow-draft at $t / T=0.25$, and maximum bow-draft at $t / T=0.75$, which is in accordance with the actual navigation constitution. Fig. 28 shows a comparison of wave contours between the original hull and optimized hull-B. It can be found that the wave elevation pattern at the forward shoulder for the optimized hull-B also reduces by the new bow shape comparing with the original hull. 


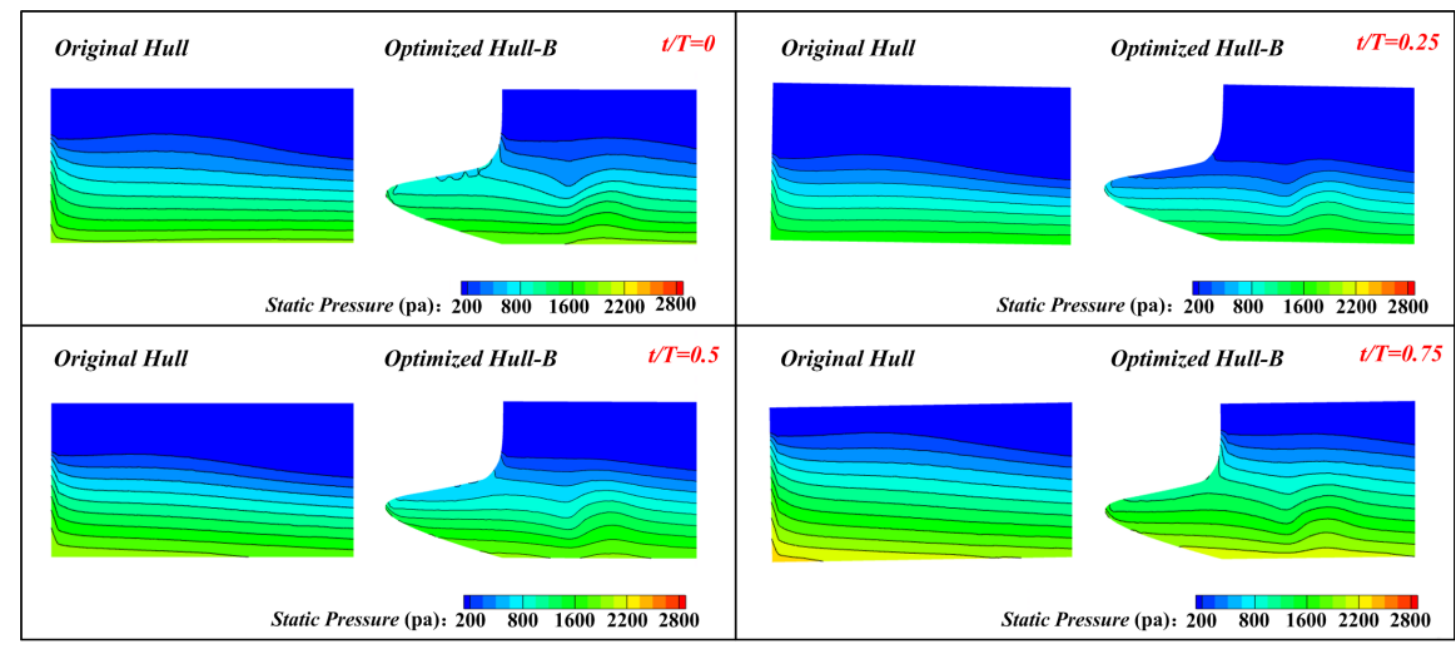

Fig. 27. Comparison of surface pressure in an encounter period

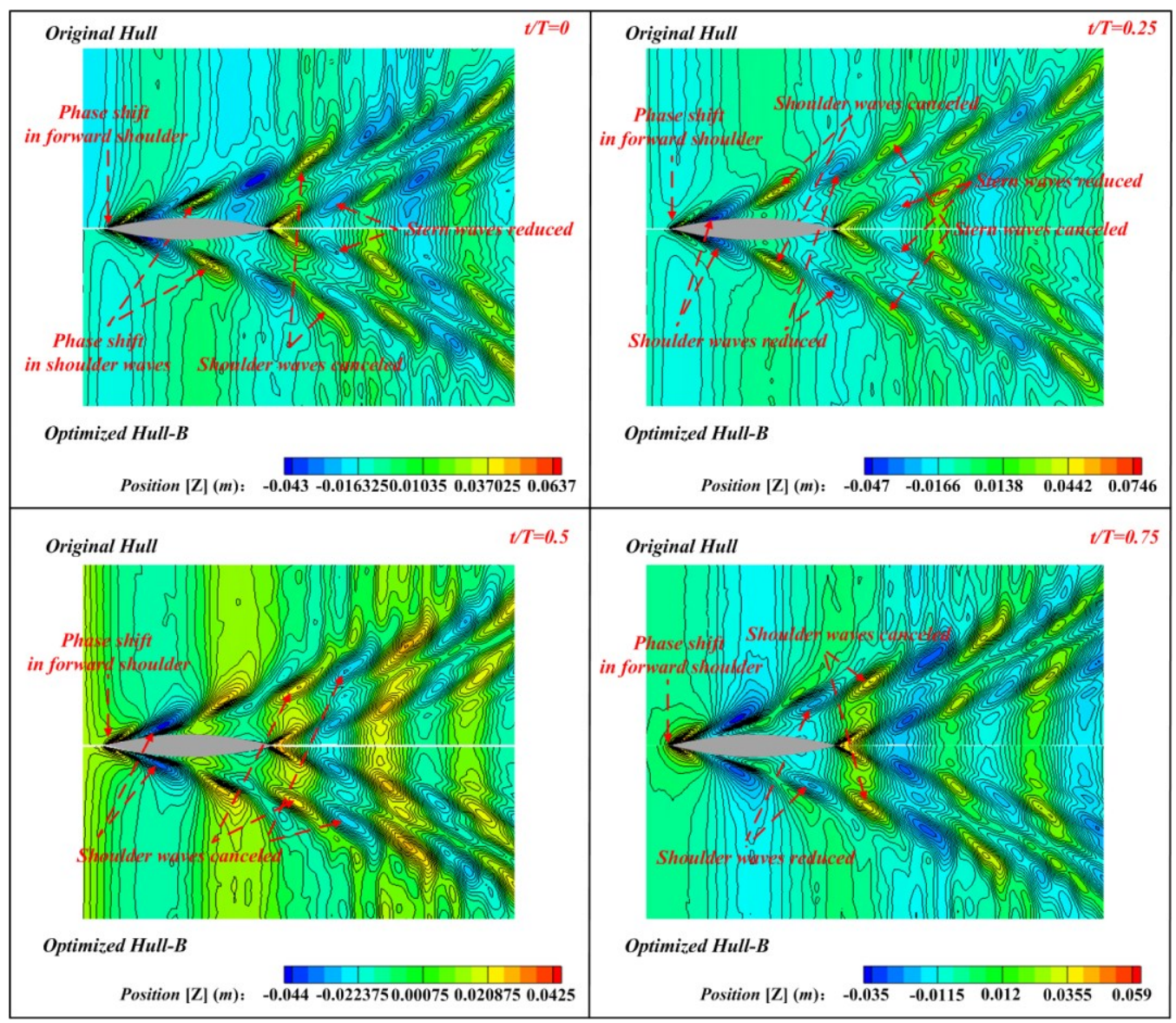

Fig. 28. Details of the free surface wave contours in an encounter period

\section{Concluding remarks}

A practical and useful ship hull form optimization framework has been presented in this paper. The numerical optimizations for the DTMB5512 and the WIGLEY III hulls were carried out in waves by changing the bow of the original hull in order to improve the resistance performance. 
Firstly, the numerical wave tank was built, and the RANS solvers were used for predicting the total resistance and the heave and pitch responses of a model-scale model in waves. The numerical modeling, wave model, time step selection, and mesh generation were all presented above. The total resistance coefficients were under predicted by $2.41 \%$ and $7.97 \%$ for DTMB5512 case and WIGLEY III case, respectively.

Next, in order to build the optimization framework, the geometry regeneration method and the optimizer are also detailed in this paper. Optimization was conducted at design speed with four design variables, respectively. The numerical simulations were carried out using the CFD software package STAR-CCM+. The new hull form with improved resistance performance can be designed through the optimization framework presented in this paper. After the completion of the optimization, the best two hull forms were obtained, and these two hull forms can decrease the total resistance by $3.71 \%$ and $4.41 \%$, respectively. The key results can be listed as follows:

1. For the two cases, the geometries of the two hulls were designed using ASD technique. This is a very practical method of geometry regeneration. At the same time, due to the few variables involved, it can also improve the optimization efficiency.

2. For the two hull form optimization cases, the hybrid optimization algorithm achieved a better hull form for resistance reduction compared with the original NLPQL algorithm. It can be demonstrated that the selection of the initial point is very important for obtaining the global optimal solution in a non-linear space.

3. The appropriate bow shape is of benefit for reducing resistance and the results also indicate the feasibility of the optimization framework for hull form optimization and provide a good starting point for the design of a new ship.

The optimization framework in this paper was presented for a single velocity with a single wave condition. Further studies will focus on a multi-objective optimization, including resistance, seakeeping and stability, to identify a ship with even better seakeeping characteristics.

\section{Acknowledgements}

The study in this paper was carried out as part of the research project: Research on Hull Form Optimization Design of Minimum Resistance Based on the Rankine Source Method and The Optimization Research of the Ship Hydrodynamic Performance Based on SBD technique. The authors gratefully acknowledge the financial support of the National Science Foundation of China (No.51009087), as well as the National Science Foundation of Shanghai (No.14ZR1419500). Results were obtained using the EPSRC funded ARCHIE-WeST High Performance Computer (www.archie-west.ac.uk). EPSRC grant no. EP/K000586/1.

\section{Reference}

Bales, N.K., (1980). Optimizing the seakeeping performance of destroyer-type hulls. 13th Symp. of Naval Hydro.,Japan.

Carrica, P.M., Wilson, R.V., Noack, R.W., Stern, F. (2007). Ship motions using single-phase level set with dynamic overset grids. Computers \& Fluids, 36(9):1415-1433.

Carrica, P.M., Fu, H., Stern, F. (2011). Computations of self-propulsion free to sink and trim and of motions in head waves of the KRISO container ship (KCS) model. Applied Ocean Research, 33(4):309-320.

CD-Adapco, (2014). User guide STAR-CCM+ Version 9.0.2.

Choi, J. and Sung, B.Y. (2009). Numerical simulations using momentum source wave-maker applied to RANS equation model. Coastal Engineering, 56(10):1043-1060.

Gammon, M.A. (2011). Optimization of fishing vessels using a Multi-Objective Genetic Algorithm. Ocean Engineering, 38(10):1054-1064.

Grigoropoulos, G.J., Chalkias, D.S. (2010). Hull-form optimization in calm and rough water. Computer-Aided Design, 42(11):977-984.

Gui, L., Longo, J., Metcalfet, B., Shao, J., Stern, F. (2001). Forces, moment, and wave pattern for surface combatant in regular head waves Part I. Measurement systems and uncertainty assessment. Experiments in fluids, 31(6):674-680. 
Gui, L., Longo, J., Metcalfet, B., Shao, J., Stern, F. (2002). Forces, moment, and wave pattern for surface combatant in regular head waves Part II. Measurement results and discussions. Experiments in fluids, 32(1):27-36.

Hirt, C. W., Nichols, B. (1981). D. Volume of fluid method for the dynamics of free boundaries. Comp. Phys, 39(1): 20-225.

Huang, F., Wang, L., Yang, C. (2015). Hull Form Optimization for Reduced Drag and Improved Seakeeping Using a Surrogate-Based Method. International Ocean \& Polar Engineering Conference: USA.

International Towing Tank Conference (ITTC), 2011b. Practical guidelines for ship CFD applications. In: Proceedings of the 26th ITTC. Available from: 〈http://ittc. sname.org/CD\%202011/pdf\%20Procedures\%202011/7.5-03-02-03.pdf (accessed 01.02.14).

Irvine, M., Longo, J., Stern, F. (2008). Pitch and heave tests and uncertainty assessment for a surface combatant in regular head waves. Journal of Ship Research, 52(2):146-163.

J.M.J.JOURNÉE. Experiments and calculations on 4 Wigley hull forms in head waves. Delft University of Technology, 1992.

Kim, H., Yang, C., Noblesse, F. (2010). Hull form optimization for reduced resistance and improved seakeeping via practical designed-oriented CFD tools. Conference on Grand Challenges in Modeling \& Simulation, 38(13):1439-1447.

Kükner, A., Sariöz, K. (1995). High speed hull form optimisation for seakeeping. Advances in Engineering Software, 22(3):179-189.

Mahmood, S., Huang, D.B. (2012). Computational fluid dynamics based bulbous bow optimization using a genetic algorithm. Journal of Marine Science and Application, 11(3):286-294.

Morris, M.D. and Mitchell, T.J. (1995). Exploratory designs for computational experiments. Journal of Statistical Planning and Inference, 43:381-402.

Nie, Q.B., Cai, T., Wang, N. (2016). Application of improved ant colony algorithm in resource allocation of cloud computing. Computer Engineering and Design, 37(8):2016-2020. (In Chinese)

Özdemir, Y.H., Çoşgun, T., Doğrul, A., Barlas, B. (2016). A numerical application to predict the resistance and wave pattern of KRISO container ship. Brodogradnja, 67(2):47-65.

Özüm, S., Şener, B., Yilmaz, H. (2005). A parametric study on seakeeping assessment of fast ships in conceptual design stage. Ocean Engineering, 38(13):1439-1447.

Park, D.W., Choi, H.J. (2013). Hydrodynamic hull form design using an optimization technique. Journal of the Society of Naval Architects of Korea, 3(1):1-9.

Patankar, S.V., Spalding, D.B. (1972). A calculation procedure for heat, mass and momentum transfer in three-dimensional parabolic flows. International Journal of Heat and Mass Transfer, 5(15): 1787-1806.

Park, J.H., Choi, J.E., Chun, H.H. (2015). Hull-form optimization of KSUEZMAX to enhance resistance performance. International Journal of Naval Architecture and Ocean Engineering, $7: 100-114$.

Kavli, H.P., Oguz, E., Tezdogan, T. (2017). A comparative study on the design of an environmentally friendly RoPax ferry using CFD. Ocean Engineering, 137, 22-37.

Qi, P. and Wang, Y.X. (2003). 3-d numerical-wave-tank technology and its application. Journal of Dalian University of Technology, 43(6):825-830. (In Chinese)

Rao, S.S., (1999). Engineering optimization: theory and practice. $3^{\text {rd }}$ Ed. New Jersey: Wiley-Interscience.

Serani, A., Fasano, G., Liuzzi, G., Lucidi, S., Iemma, U., Campana, E.F., Stern, F., Diez, M. (2016). Ship hydrodynamic optimization by local hybridization of deterministic derivative-free global algorithms. Applied Ocean Research, 59:115-128.

Shen, Z.R., Ye, H.X., Wan, D.C. (2014). URANS simulations of ship motion responses in long-crest irregular waves. Journal of Hydrodynamics, 26(3):436-446.

Sun, J., Tu, H., Chen, Y., Xie, D., Zhou, J. (2016). A study on trim optimization for a container ship based on effects due to resistance. Journal of Ship Research, 60(1):30-47. 
Sun, Z.X., Song, J.J., An, Y.R. (2010). Optimization of the head shape of the CRH3 high speed train. Science China of Technological Sciences, 53:3356-3364.

Schittkowski, K. (1985). NLPQL: a fortran subroutine solving constrained nonlinear programming problems. Annals of Operations Research, 5(6):485-500.

Tezdogan, T., Demirel, Y.K., Kellett, P., Khorasanchi, M., Incecik, A., Turan, O. (2015). Full-scale unsteady RANS CFD simulations of ship behaviour and performance in head seas due to slow steaming. Ocean Engineering, 97:186-206.

Tungadio, D.H., Jordaan, J.A., Siti, M.W. (2016). Power system state estimation solution using modified models of PSO algorithm: Comparative study. Measurement, 92:508-523.

Yakhot, V., Orszag, S.A., (1986). Renormalization group analysis of turbulence: I. Basic theory. Journal of Scientific Computing, 1 (1), 1-51.

Yang, Z., Lin, Q., Guo, Z.Q., Ma, W.J. (2015). Numerical simulation research on wet deck bow slam for large high speed catamarans. Journal of Huazhong University of Science and Technology, 43(6):42-47. (In Chinese)

Yousefi, R., Shafaghat, R., Shakeri, M. (2014). High-speed planing hull drag reduction using tunnels. Ocean Engineering, 84:54-60.

Wang, S., Su, Y.M., Pang, Y.J., Liu, H.X., (2014). Numerical study on longitudinal motions of a high-speed planing craft in regular waves. Journal of Harbin Engineering University, 35(1):45-52. (In Chinese)

Zhang, B.J. (2012). Optimization design of hull lines based on hybrid optimization algorithm. Journal of Shanghai Jiaotong University, 46(8):1238-1242. (In Chinese)

Zhang, B.J., Zhang, Z.X. (2015). Research on theoretical optimization and experimental verification of minimum resistance hull form based on Rankine source method. International Journal of Naval Architecture \& Ocean Engineering, 7(5):785-794. 